Polymer Journal Vol. 1, No. 6, pp 736-741 (1970)

\title{
Reactivity of Trimethylsilyl Vinyl Ether in Cationic Copolymerization and Some Related Reactions
}

\author{
Shun-ichi Nozakura, Masami Kitamura, and Shunsuke Murahashi \\ Faculty of Science, Osaka University, Toyonaka, Osaka, Japan.
}

(Received on August 20, 1970)

\begin{abstract}
In order to ascertain the relative reactivity of trimethylsilyl vinyl ether among vinyl ethers, cationic copolymerizations were carried out. Relative rates in bromine addition to the double bonds of vinyl ethers and relative stabilities of the iodine complexes were also studied as related reactions.

$n-, i$, and $t$-Butyl vinyl ethers were increasingly reactive in this order in all of these reactions. The reactivity of trimethylsilyl vinyl ether was between the $i$ - and $t$-butyl vinyl ethers in both the copolymerization and iodine-complex formations, and at its lowest in the bromine addition.

The reactivities of trimethylsilyl vinyl ether are rationalized by considering the contribution of the inductive effect of the trimethylsilyl group as well as the various degrees of participation of silicon $3 d$ orbital in stabilizing the transition states.

KEY WORDS Trimethylsilyl Vinyl Ether/Vinyl Ether/Cationic

Copolymerization / Bromine Addition / Iodine Complex / Relative

Reactivity / Silicon $3 d$ Orbital /
\end{abstract}

It is known that trimethylsilyl vinyl ether (VOSi) is similar in many respects to the corresponding carbon analogue, vinyl ether, and that VOSi is unique because of the lability of the $\mathrm{Si}-\mathrm{O}$ bond toward hydrolysis. VOSi polymerizes by cationic mechanisms ${ }^{1}$, while it scarcely homopolymerizes by radical mechanisms, but does copolymerize with various vinyl monomers by radical mechanisms ${ }^{2}$, thus closely resembling vinyl ethers.

The object of the present paper is to locate VOSi among vinyl ethers and to discover how the difference between silicon and carbon reflects on the reactivity of VOSi and vinyl ethers. In our previous paper $^{3}$, monomer reactivity ratios, $Q$, and $e$ values of VOSi were determined in comparison with $i$-butyl vinyl ether ( $i$-BVE) using acrylonitrile, methacrylonitrile, vinyl acetate, and methyl methacrylate as comonomers in radical copolymerizations. We could not recognize any appreciable difference between the two: for VOSi, $Q=0.018, e=-2.39$; for $i$-BVE, $Q=0.019, e=-2.11$. Since the comparison of reactivity by radical copolymerizations appears to be rather insensitive, we attempted in the present work the cationic copolymerizations of VOSi with $n$-, $i$-, and $t$-BVE in order to refine the comparison.

Further comparisons were made using the addition reaction of bromine to the double bond and the complex formation reaction with iodine. The bromine addition is related to cationic polymerization as one of the typical electrophilic additions to double bonds. The primary step of these addition reactions may be considered the $\pi$-complex formation of the reactant with the double bond. The $\pi$-complex formation of iodine with monomers was studied from this point of view.

\section{EXPERIMENTAL}

\section{Materials}

VOSi was prepared according to the method of Nesmeyanov ${ }^{4}$ from mercuridiacetaldehyde and trimethylchlorosilane, bp $74.3-74.5^{\circ} \mathrm{C}$.

$n$ - and $i$-BVEs were commercial reagents, washed with aqueous sodium hydroxide, dried with sodium hydroxide, refluxed over sodium metal for $8 \mathrm{hrs}$ and distilled.

$t$-BVE was prepared by vinylation of $t$-butanol in the presence of potassium $t$-butoxide at $150^{\circ} \mathrm{C}$ under the acetylene pressure of $20 \mathrm{~atm}$. The crude product was refluxed over sodium and 
distilled, bp $76^{\circ} \mathrm{C}, 62.4 \%$ yield.

Tin tetrachloride and trichloroacetic acid were purified by simple distillation of commercial products.

\section{Copolymerization}

The formation of the copolymer was confirmed by the solubility test of the product. A monomer mixture of VOSi $(0.0127 \mathrm{~mol})$ and $i$-BVE $(0.0166 \mathrm{~mol})$ in $50 \mathrm{ml}$ of toluene was copolymerized at $-78^{\circ} \mathrm{C}$ for $30 \mathrm{~min}$ using $0.468 \mathrm{mmol}$ of $\mathrm{SnCl}_{4}-\mathrm{Cl}_{3} \mathrm{CCOOH}(2: 1)$ catalyst. The mixture was quenched by the addition of methanol containing a small amount of concd $\mathrm{HCl}$ to effect at the same time the solvolysis of the VOSi units. The composition of the polymer was $62.2 \mathrm{~mol} \%$ of $i$-BVE and $37.8 \mathrm{~mol} \%$ of vinyl alcohol (from $\mathrm{C}$ and $\mathrm{H}$ analysis). $[\eta]=$ $0.413 \mathrm{~d} l / \mathrm{g}$ in acetone at $30^{\circ} \mathrm{C}$. The polymer was soluble in acetone, and insoluble in water. An artificial mixture of poly( $i$-BVE) and poly (vinyl alcohol), which was precipitated from a hot dimethylformamide solution into methanol, could be completely separated by extraction with benzene. The copolymerization product was partly soluble in benzene but the composition of the benzene-soluble part was identical in IR spectra to the benzene-insoluble fraction. The fact that the polymer did not precipitate from an acetone solution with methanol and was insoluble in water shows the absence of homopolymers of $i$-BVE and vinyl alcohol.

Monomer reactivity ratios were determined by measuring the amount of residual monomers by gas chromatography. An aliquot of the copolymerization mixture was taken at intervals through a capillary outlet tube by applying nitrogen pressure. Receiver vials contained a small amount of pyridine to stop the polymerization. Gas chromatography was performed using a column of tricresyl phosphate on celite $545(75 \mathrm{~cm})$ and Silicone DC 550 on celite 545 $(75 \mathrm{~cm})$ at $100^{\circ} \mathrm{C}$. The internal standard was cyclohexane in the case of $n$-BVE and methylcyclohexane in the case of $i$ - and $t$-BVE.

\section{Addition Reaction of Bromine}

Stoichiometry of the addition of bromine to monomers was confirmed. VOSi $(0.0146 \mathrm{~mol})$ in benzene was titrated with a carbon tetrachloride solution of bromine at $0^{\circ} \mathrm{C}$, absorbing
$0.0148 \mathrm{~mol}$ of bromine. The mixture was distilled to yield $64.8 \%$ of dibromide, bp $50^{\circ} \mathrm{C}$ $(4 \mathrm{~mm})$. The product appears not to have been sufficiently stable under these distillation conditions. A monomer mixture of VOSi $(2.00$ mmol) and $n$-BVE $(1.96 \mathrm{mmol})$ in carbon tetrachloride consumed $4.06 \mathrm{mmol}$ of bromine at $0^{\circ} \mathrm{C}$; that of VOSi $(2.00 \mathrm{mmol})$ and $i$-BVE $(1.99$ mmol) consumed $3.99 \mathrm{mmol}$ of bromine, and that of VOSi $(2.00 \mathrm{mmol})$ and $t$-BVE $(1.99 \mathrm{mmol})$ consumed $4.02 \mathrm{mmol}$ of bromine. Thus, it was confirmed that the addition reaction was quantitative without being accompanied by polymerization.

Relative rate measurement was carried out using the competitive reaction of two monomers at $0^{\circ} \mathrm{C}$ in carbon tetrachloride. A bromine solution was added dropwise to a monomer mixture, disappearance of bromine color taking place instantaneously. At intervals the residual monomers were analyzed by gas chromatography using toluene as an internal standard (Silicone DC 550 on celite $545,75 \mathrm{~cm}, 50^{\circ} \mathrm{C}$ ).

\section{Complex Formation of Iodine with Monomers}

Equilibrium constants of the complex formation between iodine and a monomer was measured according to the method of Higashimura and others ${ }^{5}$ using a Hitachi 139 specrophotometer.

\section{RESULTS}

\section{Copolymerization}

Table I shows an example of the analysis of residual monomer concentrations during a copolymerization. From this data correlation curves between two monomer concentrations are drawn as illustrated in Figure 1. We obtain $\mathrm{d}\left[\mathrm{M}_{1}\right] / \mathrm{d}\left[\mathrm{M}_{2}\right]$ as a slope of these correlation curves. The values of $\left[\mathrm{M}_{1}\right] /\left[\mathrm{M}_{2}\right]$ and the corresponding values of $d\left[M_{1}\right] / d\left[M_{2}\right]$ give a FinemanRoss plot as shown in Figure 2. Monomer reactivity ratios thus determined are summarized in Table II.

Reciprocal $r_{1}$ shows the relative reactivities of monomers toward the propagating VOSi cation, indicating the order

$$
t \text {-BVE }>\text { VOSi }>i \text {-BVE } \approx n \text {-BVE }
$$

in both the solvents of toluene and nitroethane. 
S. Nozakura, M. Kitamura and S. Murahashi

Table I. Copolymerization of VOSi $\left(\mathrm{M}_{1}\right)$ and $t$-VBE $\left(\mathrm{M}_{2}\right)$

In toluene, $\left[\mathrm{M}_{1}\right]=0.0126 \mathrm{~mol} / l,\left[\mathrm{M}_{2}\right]=0.0270 \mathrm{~mol} / l$, $[\mathrm{Cat}]=0.936 \mathrm{mmol} / \mathrm{l}$

\begin{tabular}{ccc}
\hline Time, $\min$ & {$\left[\mathrm{M}_{1}\right], \mathrm{mol} / l$} & {$\left[\mathrm{M}_{2}\right], \mathrm{mol} / l$} \\
\hline 1.90 & 0.0119 & 0.0237 \\
3.65 & 0.0115 & 0.0225 \\
16.00 & 0.0091 & 0.0137 \\
22.50 & 0.0081 & 0.0113 \\
35.58 & 0.0066 & 0.0074 \\
\hline
\end{tabular}

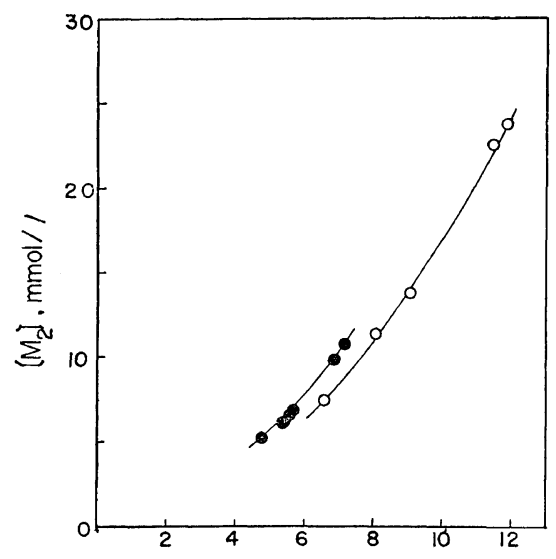

$\left[\mathrm{M}_{\mathrm{l}}\right], \mathrm{mmol} / /$

Figure 1. Copolymerization of VOSi $\left(\mathrm{M}_{1}\right)$ and $t$ $\operatorname{BVE}\left(\mathrm{M}_{2}\right)$ at $-78^{\circ} \mathrm{C}$ : $\bigcirc$, in toluene; $\odot$, in nitroethane.
In nitroethane, $\left[\mathrm{M}_{1}\right]=0.0079 \mathrm{~mol} / \mathrm{l}, \quad\left[\mathrm{M}_{2}\right]=0.0130$ $\mathrm{mol} / l$, [Cat $]=0.936 \mathrm{mmol} / l$

\begin{tabular}{ccc}
\hline Time, min & {$\left[\mathrm{M}_{1}\right], \mathrm{mol} / l$} & {$\left[\mathrm{M}_{2}\right], \mathrm{mol} / l$} \\
\hline 4.35 & 0.0072 & 0.0107 \\
5.83 & 0.0069 & 0.0098 \\
17.25 & 0.0057 & 0.0068 \\
22.00 & 0.0056 & 0.0065 \\
30.08 & 0.0054 & 0.0061 \\
40.50 & 0.0048 & 0.0052
\end{tabular}

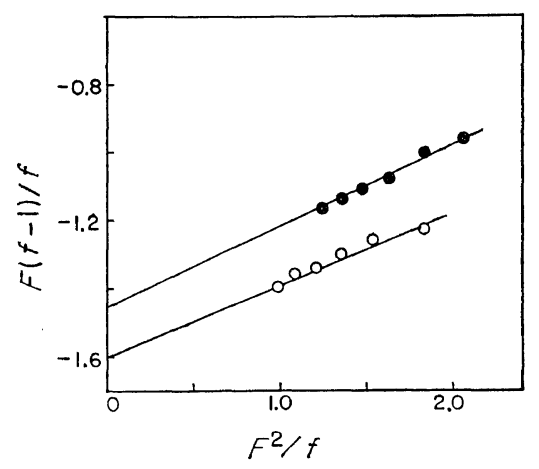

Figure 2. Fineman-Ross plots for the copolymerization of $\operatorname{VOSi}\left(\mathrm{M}_{1}\right)$ and $t$-BVE $\left(\mathrm{M}_{2}\right)$ at $-78^{\circ} \mathrm{C}$ : $\bigcirc$, in toluene; 0 , in nitroethane.

Table II. Monomer reactivity ratios in the copolymerization of VOSi $\left(\mathrm{M}_{1}\right)$ and $\operatorname{BVE}\left(\mathrm{M}_{2}\right)$

\begin{tabular}{cccccc}
\hline \multirow{2}{*}{$\mathrm{M}_{2}$} & \multicolumn{2}{c}{ In toluene } & & \multicolumn{2}{c}{ In nitroethane } \\
\cline { 2 - 5 } & $r_{1}$ & $r_{2}$ & & $r_{1}$ & $r_{2}$ \\
\hline$t$-BVE & $0.21 \pm 0.04$ & $1.60 \pm 0.04$ & & $0.24 \pm 0.06$ & $1.45 \pm 0.09$ \\
$i$-BVE & $1.37 \pm 0.06$ & $0.34 \pm 0.02$ & & $1.08 \pm 0.05$ & $0.35 \pm 0.03$ \\
$n$-BVE & $1.40 \pm 0.10$ & $0.18 \pm 0.03$ & & $1.04 \pm 0.09$ & $0.32 \pm 0.09$ \\
\hline
\end{tabular}

Magnitude of the difference in relative reactivity was smaller in nitroethane than in toluene, presumably because the higher reactivity of the propagating cation in the more polar medium resulted in less selectivity to monomers.

\section{Addition of Bromine}

The rate of addition of bromine to olefin, in general, can be expressed by eq $1, n$ being 1 or 2 depending on the reaction conditions. ${ }^{6}$

$$
-\mathrm{d}[\text { Olefin }] / \mathrm{d} t=k\left[\text { Olefin] }\left[\mathrm{Br}_{2}\right]^{n}\right.
$$

In the reaction conditions of competitive reaction, eq 1 can be applied to both monomers with the same $n$. Therefore, we obtain eq 2 .

$$
\frac{\mathrm{d}[\mathrm{VOSi}]}{\mathrm{d}[\mathrm{BVE}]}=\frac{k_{\mathrm{VOSi}}[\mathrm{VOSi}]}{k_{\mathrm{BVE}}[\mathrm{BVE}]}
$$

Integration gives eq 3 . 


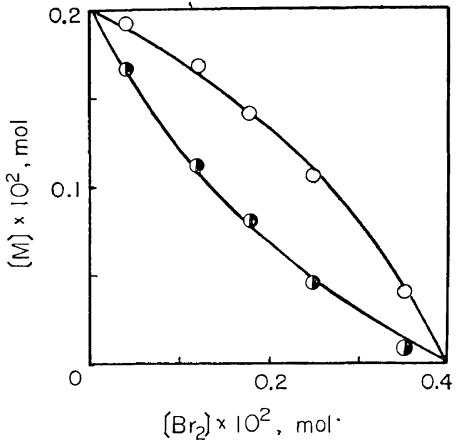

Figure 3. Addition of bromine to a mixture of VOSi and $t$-BVE in carbon tetrachloride at $0^{\circ} \mathrm{C}$ : $\bigcirc$, VOSi; $1, t$-BVE.

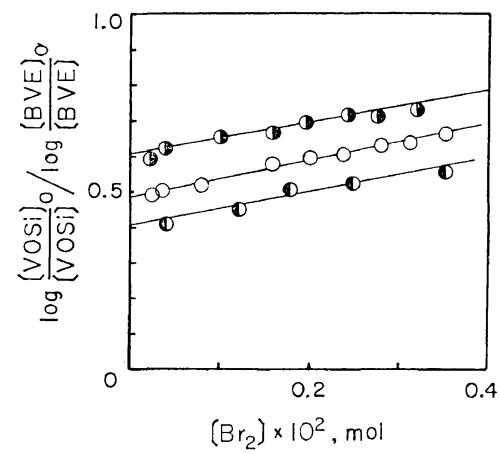

Figure 4. Addition of bromine to a mixture of VOSi and vinyl ethers in carbon tetrachloride at $0^{\circ} \mathrm{C}$ :,$n$-BVE; $\bigcirc, i$-BVE;,$t$-BVE.

$$
\frac{\log \left([\mathrm{VOSi}]_{0} /[\mathrm{VOSi}]\right)}{\log \left([\mathrm{BVE}]_{0} /[\mathrm{BVE}]\right)}=\frac{k_{\mathrm{VOSi}}}{k_{\mathrm{BVE}}}
$$

Disappearance of the monomers during the bromide addition is illustrated in Figure 3. The left side of eq 3 was calculated from this data and plotted against the amount of added bromine in Figure 4, showing a slight linear drift during the entire reaction. One of the reasons for the drift might be the change in dielectric properties of the reaction medium. Extrapolated values to zero bromine addition were taken as relative velocity constants in eq 3 . Figure 4 indicates the following order of reactivity,

$$
\begin{aligned}
& t \text {-BVE }>i \text {-BVE }>n \text {-BVE }>\text { VOSi } \\
& \begin{array}{llll}
2.48 & 2.07 & 1.66 & 1.00
\end{array}
\end{aligned}
$$

\section{Iodine-Complex Formation}

All the vinyl ethers including VOSi showed absorptions at around $275 \mathrm{~m} \mu$ upon mixing with

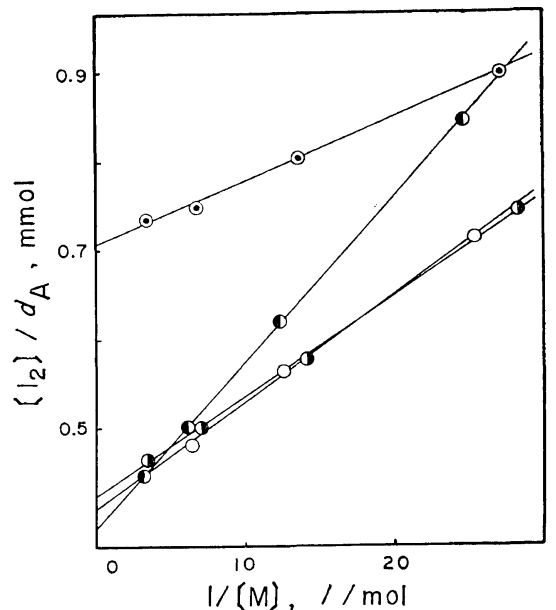

Figure 5. Complex formation between iodine and monomers in isooctane at room temperature: $D$, $n$-BVE; $\bigcirc, i$-BVE; $\odot, t$-BVE; $($ VOSi.

Table III. Equilibrium constants of iodine-vinyl ether complexes in isooctane at room temperature

\begin{tabular}{ccc}
\hline Monomer & $\varepsilon_{\mathrm{A}} K_{\mathrm{A}} \times 10^{-4}, l^{2} / \mathrm{mol}^{2}$ & $K_{\mathrm{A}}+K_{\mathrm{B}}, l / \mathrm{mol}$ \\
\hline$t$-BVE & 14.1 & 99.6 \\
VOSi & 9.0 & 37.7 \\
$i$-BVE & 8.2 & 34.0 \\
$n$-BVE & 5.4 & 21.0 \\
\hline
\end{tabular}

iodine in isooctane. This absorption is known to be due to the complex formation between iodine and the double bond of vinyl ethers. ${ }^{5}$ Absorbance at this maximum $d_{A}$, iodine concentration $\left[\mathrm{I}_{2}\right]$ and monomer concentration $[\mathrm{M}]$ provide information about the equilibrium according to eq 4 , where $\varepsilon_{\mathrm{A}}$ denotes the absorption coefficient of the complex, and $K_{\mathrm{A}}$ and $K_{\mathrm{B}}$ denote equilibrium constants

$$
\frac{\left[\mathrm{I}_{2}\right]}{d_{\mathrm{A}}}=\frac{1}{\varepsilon_{\mathrm{A}} K_{\mathrm{A}}[\mathrm{M}]}+\frac{K_{\mathrm{A}}+K_{\mathrm{B}}}{\varepsilon_{\mathrm{A}} K_{\mathrm{A}}}
$$

of the iodine-complex formation with the double bond and with the ether oxygen of vinyl ethers, respectively. Experimental plots are shown in Figure 5 and numerical results are listed in Table III. The magnitude of $\varepsilon_{\mathrm{A}}$ might be considered nearly constant among these vinyl ethers, and so relative stabilities of the complex as judged from the $\varepsilon_{\mathrm{A}} K_{\mathrm{A}}$ values are in the order, 


\section{S. Nozakura, M. Kitamura and S. Murahashi}

$$
t \text {-BVE }>\text { VOSi }>i \text {-BVE }>n \text {-BVE }
$$

This order is supported from the values of the overall equilibrium constants $K_{\mathrm{A}}+K_{\mathrm{B}}$.

\section{DISCUSSION}

Relative reactivities of VOSi and $n$-, $i$-, and $t$-BVE were compared in the cationic copolymerization, bromine addition, and equilibrium constants of the iodine-complex formation. The results are summarized in Table IV along with the NMR methylene geminal coupling constants of vinyl groups ${ }^{2,7}$ and the electron-releasing tendency of the alkyl groups of vinyl ethers. ${ }^{8}$

As far as butyl vinyl ethers are concerned, the relative reactivities in the copolymerization, the bromine addition, and the iodine-complex formation are always in the order $t$-BVE $>i$ BVE $>n$-BVE. VOSi is located between $t$-BVE and $i$-BVE in the copolymerization and the iodine-complex formation. The situation is the same in geminal coupling constants of the

Table IV. Relative reactivities of VOSi, $n-, i$, and $t$-BVE

\begin{tabular}{|c|c|c|}
\hline Copolymerization & \multicolumn{2}{|c|}{$t-\mathrm{BVE}>\mathrm{VOSi}>i-\mathrm{BVE} \approx n-\mathrm{BVE}$} \\
\hline Bromine addition & $t$-BVE $>$ & $i-\mathrm{BVE}>n-\mathrm{BVE}>\mathrm{VOSi}$ \\
\hline Iodine complex & \multicolumn{2}{|c|}{$t-\mathrm{BVE}>\mathrm{VOSi}>i-\mathrm{BVE}>n-\mathrm{BVE}$} \\
\hline NMR, $J_{\text {gem }}{ }^{2,7}$ & $\begin{array}{cc}t-\mathrm{BVE} & \mathrm{VOSi} \\
0.1 & 0.7\end{array}$ & $\begin{array}{cc}i \text {-BVE } & n \text {-BVE } \\
1.7 & 1.8\end{array}$ \\
\hline Electron-releasing effect of $R^{8}$ & $\mathrm{VOSi}>t$-BVE $\quad>$ & $i$-BVE $>n-\mathrm{BVE}$ \\
\hline
\end{tabular}

methylene group. Although the relation between the NMR spectra of alkyl vinyl ether and the reactivity in the cationic copolymerization is complicated by the presence of the inductive, resonance and steric effects of the alkyl group, ${ }^{9}$ we used the geminal coupling constants as a measure of the electron-releasing power of the alkyl group. ${ }^{7}$ In the bromine addition VOSi is the least reactive.

Reactivities of butyl vinyl ethers may be rationalized by the difference in inductive effect of alkyl groups, the most electron-releasing $t$ butyl giving the highest reactivity. The inductive effect of the trimethylsilyl group is known to be more electron-releasing than $t$-butyl. ${ }^{8}$ If the inductive effect is a predominant factor in determining reactivity, VOSi should be the most reactive among the monomers tested. However, a silicon atom will use a $3 d$ orbital to form a $p \pi-d \pi$ bond with an oxygen atom, withdrawing electrons from the oxygen toward the silicon atom. This electron-withdrawing effect will offset the inductive effect of the trimethylsilyl group. The net effect of the trimethylsilyl group might have located VOSi between $t$ - and $i$-BVE in the copolymerization, the iodine-complex formation, and NMR.

In the bromine addition, the participation of $3 d$ orbital of silicon in the stabilization of the transition state might be greater than in other reactions. If we take into consideration the fact that the location of VOSi in the copolymerization and the iodine-complex formation is similar to the situation in the NMR spectra of the monomers, the transition state in the copolymerization and the complexed state with iodine will be electronically close to the initial state as, for example, in I. On the other hand, the transition state in the bromine addition will be relatively remote from the initial state as, for example, in II.

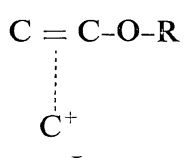

I

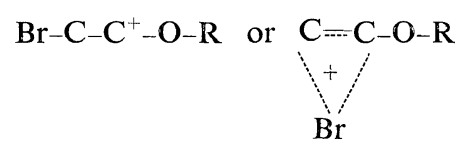

II
In conclusion, the relative reactivity of VOSi closely resembles that of vinyl ethers. The relative location of VOSi among vinyl ethers varies with the type of reaction and may depend upon the combinations of the inductive and the resonance effect of the trimethylsilyl group.

\section{REFERENCES}

1. S. Murahashi, S. Nozakura, and M. Sumi, $J$. Polym. Sci., Part B, 3, 245 (1965).

2. S. Murahashi, S. Nozakura, M. Sumi, and R. 
Ohno, ibid., 4, 187 (1966).

3. R. Ohno, S. Nozakura, and S. Murahashi, Kobunshi Kagagu (Chem. High Polymers), 25, 188 (1968).

4. A. N. Nesmeyanov, I. F. Lutsenko, and V. A. Brotsev, Dokl. Akad. Nauk SSSR, 128, 515 (1959).

5. T. Higashimura, N. Kano, T. Yonezawa, K. Fukui, and S. Okamura, Nippon Kagaku Zasshi (J. Chem. Soc, Japan, Pure Chem. Sect.), 81, 550 (1960).
6. J. Hine, "Physical Organic Chemistry," 2nd ed, McGraw-Hill Book Co. Inc., New York, N.Y., 1962, p 217.

7. J. Feeney, A. Ledwith, and L. H. Sutcliffe, $J$. Chem. Soc., 1962, 2021.

8. L. H. Sommer and J. Rockett, J. Amer. Chem. Soc., 73, 5130 (1951).

9. H. Yuki, K. Hatada, and M. Takeshita, J. Polym. Sci., Part A-1, 7, 667 (1969). 